\title{
Medium induced radiation in multiparton splittings
}

\section{Fabio Domínguez}

Universidade de Santiago de Compostela

E-mail: fabio.dominguez@usc.es

\section{Carlos A. Salgado}

Universidade de Santiago de Compostela

E-mail: carlos.salgadodusc.es

\section{Víctor Vila*}

Universidade de Santiago de Compostela

E-mail: victor.vila@usc.es

\begin{abstract}
We study the color coherence phenomenon starting from the original quark-antiquark antenna laboratory. Firstly, we add a new in-medium hard gluon emission, and after that another one emitted outside it. Describing the decoherence of the $q \bar{q}$ pair through the survival probability, we extract some interesting interpretations in the large- $N_{c}$ limit. With the purpose of considering a more realistic setting, we address a similar configuration in which a quark-gluon antenna with finite formation time propagates through the medium, and the gluon emits an extra soft gluon afterwards. Finally, we shed light on the role of color coherence in these configurations.
\end{abstract}

12th International Workshop on High-pT Physics in the RHIC/LHC Era

2-5 October, 2017

University of Bergen, Bergen, Norway

${ }^{*}$ Speaker. 


\section{Jet substructure}

The study of jet substructure is becoming one of the most interesting ways to explore the QGP properties in heavy-ion collissions at the LHC. This new tool provides us more sophisticated observables that could shed light on the details of the jet propagation through the dense medium formed in the collisions. Nevertheless, some questions remain to be answered to achieve a consistent understanding of the generic mechanism that is expected to lead jet modifications and energy loss, and hence a better characterization of the medium properties, which plays a fundamental role in addressing the coherence phenomenon [1].

\section{Antenna radiation in a medium}

Addressing the simple case in which a very soft gluon taking place outside the medium is emitted from the antenna, the contribution to the medium-induced radiation can be easily computed from the diagrams of Fig. 1. The spectrum is a modification of the one computed for the vacuum case [2]

$$
d N=\frac{d \omega}{\omega} \frac{d \Omega}{2 \pi} \frac{\alpha_{s} C_{F}}{2 \pi}\left[R_{q}+R_{\bar{q}}-\left(1-\Delta_{m e d}\right) 2 \mathscr{J}\right] .
$$

Here, the $q \bar{q}$ - medium interaction is described by the survival probability of the pair not to change the color configuration

$$
S \equiv 1-\Delta_{\text {med }} \equiv \frac{1}{N_{c}^{2}-1} \operatorname{Tr}\left\langle W\left(\vec{x}_{\perp}\right) W^{\dagger}\left(\vec{y}_{\perp}\right)\right\rangle
$$

where the propagation of a parton through a medium field $A_{-}\left(x_{+}, \vec{x}\right)$ is given by the Wilson lines

$$
W(\vec{x})=\mathscr{P} \exp \left[i g \int d x_{+} A_{-}\left(x_{+}, \vec{x}\right)\right] .
$$
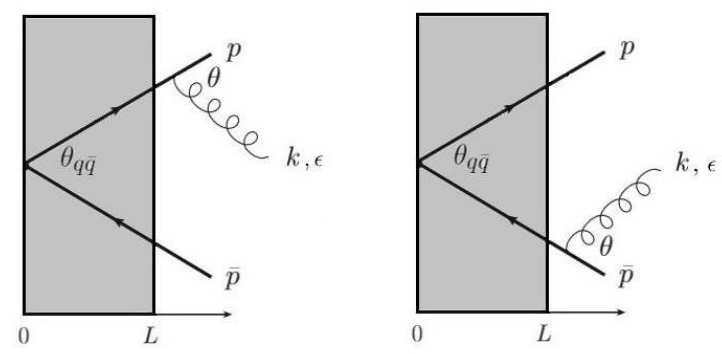

Figure 1: Out-out radiation for a very soft gluon emission outside the medium $(\omega \rightarrow 0)$.

For the particular case of a static medium of size $L$, the decoherence parameter is given - in the multiple soft scattering approximation - by

$$
\Delta_{\text {med }} \simeq 1-\exp \left[-\frac{1}{4} \hat{q} L\left(\vec{x}_{\perp}-\overrightarrow{\bar{x}}_{\perp}\right)^{2}\right]
$$

where $\hat{q}$ is the transport coefficient, containing all the information about the medium properties. 
Here we can consider two different [2,3],

1. on the one hand, when the color correlation length of the medium $\left(\simeq \frac{1}{\sqrt{\hat{q} L}}\right)$ is larger than the size of the pairs, $\Delta_{\text {med }} \rightarrow 0$, the medium cannot resolve the individual emitters, thus acting like an individual object with the total charge of the pair $\left(d N \simeq R_{q}+R_{\bar{q}}-2 \mathscr{J}\right)$.

2. on the other hand, when $\Delta_{\text {med }} \rightarrow 1$, the medium resolves the antenna and it breaks the color coherence of the pair, behaving as two independent emitters $\left(d N \simeq R_{q}+R_{\bar{q}}\right)$.

A convenient way to estimate the coherence effects is by defining a coherence time, $t_{c o h} \sim$ $\left[\frac{1}{12} \theta_{q \bar{q}}^{2} \hat{q}\right]^{1 / 3}$, such that coherence is preserved when the path lenght in the medium is smaller than $t_{\text {coh }}$.

\section{Multiple splittings}

One of the main limitations of the problem that we aimed at is that it only includes one inmedium splitting. Now, we put forward a multiple emissions problem to take in account this limitation. For this purpose, we include an additional hard gluon with the final goal of extrapolating the results to $n$-gluon emissions. As we are restricting our study to the case of very soft gluon radiation, only the out-out component needs to be computed $(\omega \rightarrow 0)$.

So, we consider the case of two hard splittings inside the medium. The direct terms, i.e., those having the same configuration both in amplitude and complex conjugate amplitude, $R_{q}$ - hard and soft gluon radiation off the quark, $R_{\bar{q}}$ - hard gluon radiation off the quark and soft gluon radiation off the antiquark - and $R_{g}$ - hard gluon radiation off the quark and soft gluon radiation off the emitted hard gluon - are proportional to the expected vacuum color factor, indicating that no medium effects appear as expected with the kinematics chosen, as we can see in the squared amplitudes written in Eq. (3.1) - corresponding to each of the figures represented just below in Fig. 2.

$$
\left|\mathscr{M}_{(1)}\right|^{2} \propto C_{F}^{2}
$$

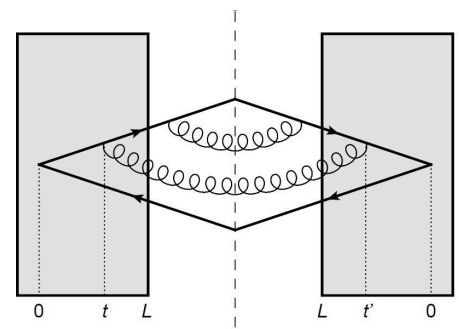

(1)

$$
\left|\mathscr{M}_{(2)}\right|^{2} \propto C_{F}^{2}
$$

$$
\left|\mathscr{M}_{(3)}\right|^{2} \propto N_{c} C_{F}^{2}
$$

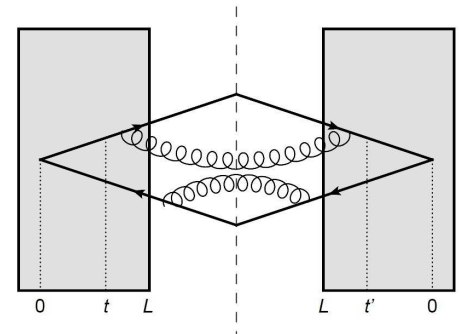

(2)

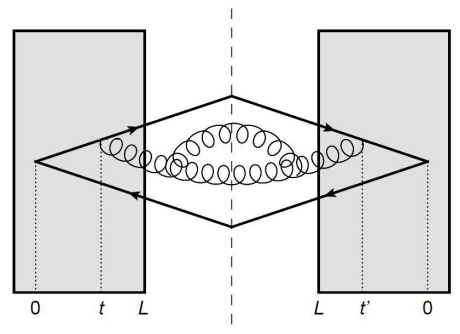

(3)

Figure 2: Direct terms for the case of three emitters. 
More interesting results are obtained in the interference terms between the diagrams (1)-(3) and (2)-(3) represented in Fig. 2, where the survival probabilities $S$ appear explicitly, providing information about the color correlation of the emitters.

The interference term between the diagrams (1) and (3) - Fig. 3 on the left side - produces the following result

$$
\mathscr{M}_{(1)} \otimes \mathscr{M}_{(3)}^{*} \propto S(t, L),
$$

where the survival probability $S$ that appear only corresponds to the $(t, L)$ region;
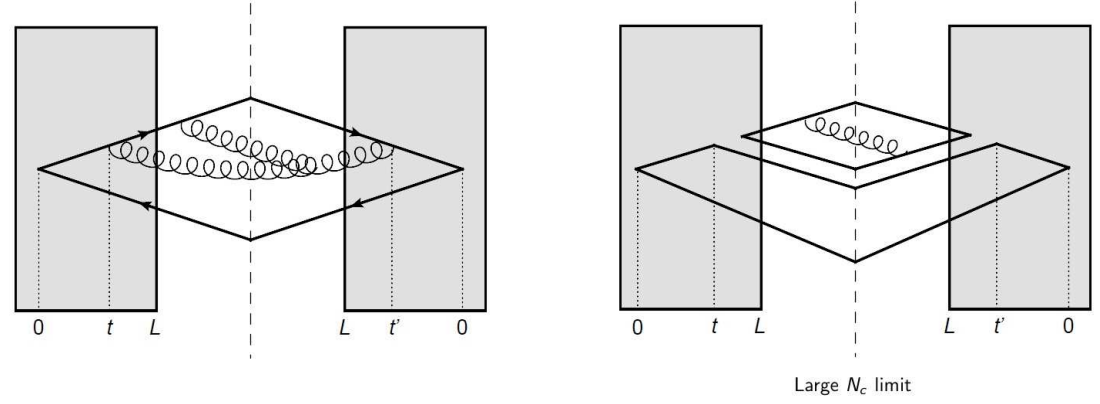

Figure 3: Interference term for the case of multiple emitters (soft gluon in amplitude).

and for the interference term between the diagrams (2) and (3) - Fig. 4 on the left side - it results

$$
\mathscr{M}_{(2)} \otimes \mathscr{M}_{(3)}^{*} \propto S(0, t) S(t, L) .
$$

In this particular case we obtain the product of the two survival probabilities - between the $(0, t)$ and $(t, L)$ regions.
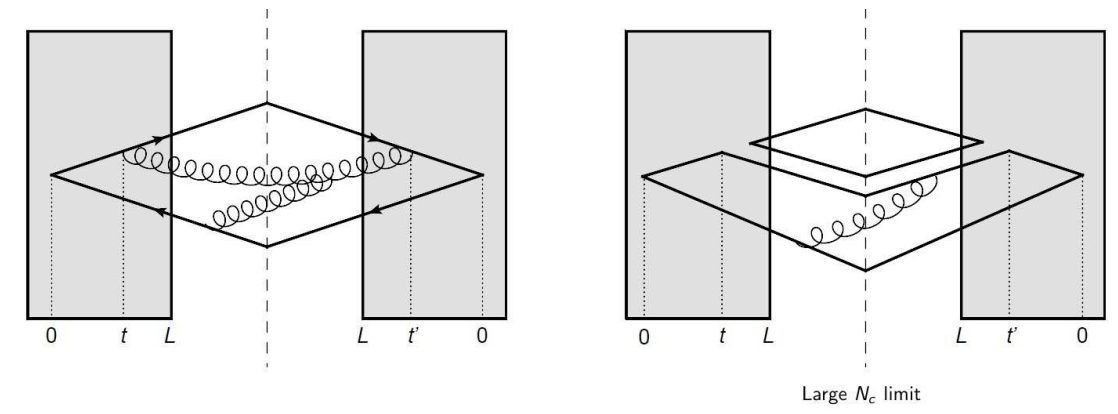

Figure 4: Interference term for the case of multiple emitters (soft gluon in conjugate amplitude).

The interpretation of the obtained results is very clear in the large- $N_{c}$ limit - see on the right side of Fig. 3 and Fig. 4. 
The degree of color coherence in the soft gluon emission is controlled by the survival probability, and it corresponds to that of the dipole from which this gluon is emitted. In Fig. 3 the dipole is formed at time $t$ during the evolution of the system, while it is produced at time 0 in the diagram of Fig. 4. Here the survival probability is the product of the two survival probabilities. Therefore, the most important result is that the general result of the original antenna is valid for each of the smaller antennas.

\section{Quark-gluon antenna through a medium}

The antenna laboratory that we addressed restrict the problem to the zero formation time case of the $q \bar{q}$ pair and eikonal propagation of the splitted partons. Let us now consider another setup to go a step further to measure the color coherence phenomena present at jet physics. Here we study a finite formation time configuration which includes the two gluon emission case - in-out configuration - of a hard quark propagating through a dense medium, as it is represented in Fig. 5.

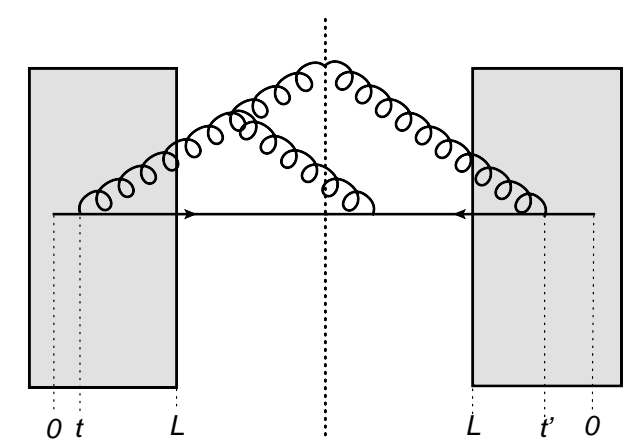

Figure 5: A hard quark propagating through a medium.

The interference term between the processes represented above - hard gluon radiation off the quark and soft gluon radiation off the emitted gluon and both hard and gluon radiation off the propagating quark - is

$$
\mathscr{M}_{1} \times \mathscr{M}_{2}^{*} \propto \frac{1}{N_{c}\left(N_{c}^{2}-1\right)}\left\langle W^{a i}(\overrightarrow{0}) W^{a i}\left(\vec{r}_{3}\right)\right\rangle_{\left(t, t^{\prime}\right)}\left\langle f^{i j c} f^{\alpha b z} W^{i \alpha}\left(\vec{r}_{3}\right) W^{j b}(\overrightarrow{0}) W^{\dagger z c}\left(\vec{r}_{3}^{\prime}\right)\right\rangle_{\left(t^{\prime}, L\right)},
$$

where $W(\overrightarrow{0})$ represents the quark propagation through the medium and $W\left(\vec{r}_{3}\right)$ and $W^{\dagger}\left(\vec{r}_{3}\right)$ the gluon propagation - through the medium - in amplitude and conjugate amplitude respectively.

Let us interpret the intermediate result obtained in Eq. (4.1). We see the emission of the gluon, taking place at time $t$ in the amplitude and time $t^{\prime}$ in the complex conjugate amplitude. In this interval, usually called formation time, the dynamics of the current dipole - $\left\langle W^{a i}(\overrightarrow{0}) W^{a i}\left(\vec{r}_{3}\right)\right\rangle_{\left(t, t^{\prime}\right)}$ - consisting of the gluon and the propagating quark is described by a path integral that incorporates the Brownian motion of the gluon in the transverse plane from $t$ to $t^{\prime}$, which comes from the accumulation of momentum from the medium. 
In the $\left(t^{\prime}, L\right)$ region, due to the simple color structure of $\left\langle f^{i j c} f^{\alpha b z} W^{i \alpha}\left(\vec{r}_{3}\right) W^{j b}(\overrightarrow{0}) W^{\dagger z c}\left(\vec{r}_{3}^{\prime}\right)\right\rangle$, we can calculate explicitly the medium average of the Wilson lines. For small formation times $t_{f}<<L$ - this diagram is suppressed by powers of $N_{c}$, recovering a result obtained in [4].

\section{Discussion and conclusions}

The color correlation of the highly energetic partons that we considered is key to understand the jet constituents' energy loss when they traverse a dense QCD medium. Proceeding as in the vacuum case, and despite the approximations taken, the $q \bar{q}$ antenna is a convenient laboratory. Considering the multiple emissions configuration, we conclude that if coherence is not preserved after the inmedium splitting, the antenna will not radiate coherently in the following emission, i.e., the original result of the antenna is valid for each of the smaller antennas. The addressed calculations and novel setups presented here provide further information to the final goal of obtaining a complete description of a QCD parton cascade.

\section{References}

[1] Y. Mehtar-Tani and K. Tywoniuk, Radiative energy loss of neighboring subjets, arxiv:1706.06047

[2] Y. Mehtar-Tani, C. A. Salgado and K. Tywoniuk, The radiation pattern of a QCD antenna in a dense medium, Phys. Lett. B 707 (2012) 156

[3] Y. Mehtar-Tani, C. A. Salgado and K. Tywoniuk, The radiation pattern of a QCD antenna in a dilute medium, JHEP 1204 (2012) 064

[4] J. P. Blaizot, F. Domínguez, E. Iancu and Y. Mehtar-Tani, Medium-induced gluon branching, JHEP 1301 (2013) 143 